\title{
TOMASZ LIS
}

Christian-Albrechts-Universität zu Kiel

t.lis@slav.uni-kiel.de

ORCID: 0000-0002-4837-9596

\author{
MARTA JANACHOWSKA-BUDYCH \\ Uniwersytet im. Adama Mickiewicza w Poznaniu \\ maja@amu.edu.pl \\ ORCID: 0000-0002-9088-5732
}

\section{Intersektionelle Filmanalyse in der fremdsprachigen Kulturdidaktik am Beispiel des MigrantInnenbildes in ausgewählten Culture-Clash-Komödien}

\section{Intersectional film analysis in foreign-language culture classes, showcasing images of migrants in selected culture-clash comedies}

\begin{abstract}
The aim of the following paper is to discuss the image of migrants presented in selected culture-clash comedies, and its didactical contribution to transcultural learning. The intersectional analysis of three film sequences conducted in the framework of university classes (seminars and workshops) on intercultural communication is described as a starting point for discussion and reflection upon the stereotyped, and popularised images of migrants as people with specific qualities and skills, certain social backgrounds, and typical patterns of behaviour. The critical analysis of such images supports transcultural learning, which is understood, among others, as bringing aspects of the development of individual as well as collective identities into question, and acknowledging heterogeneity and diversity as cultural and social enrichment.
\end{abstract}


KEYWORDS: culture didactics, transcultural learning, intersectional film analysis, interculturality, transculturality, hyperculturality, migration, image of a migrant.

SCHLÜSSELWORTE: Kulturdidaktik, transkulturelles Lernen, intersektionelle Filmanalyse, Interkulturalität, Transkulturalität, Hyperkulturalität, Migration, MigrantInnenbild.

\section{EINLEITUNG}

Als ein "per se" transkulturelles und mehrsprachiges Medium - sowohl auf der Ebene der Produktion, der Rezeption als auch der Handlung (vgl. Kepser 2015: 83) - eignet sich der Film dazu, Prozesse des transkulturellen Lernens zu initiieren und zu fördern. Mit ihrem breiten Angebot produziert, vermarktet und lanciert die Filmindustrie bestimmte Heldentypen, Verhaltensweisen und Handlungsmuster, die sich oft mit den stereotypen Vorstellungen über einige gesellschaftliche Gruppen überschneiden. Besonders interessant ist es im Fall von den in Filmen verschiedener Art dargestellten MigrantInnenbilder, die mit soziopolitischen Auffassungen der Inter-, Trans- bzw. Hyperkulturalität konfrontiert und aus deren Perspektive diskutiert werden. Dies bietet wiederum den Anlass zur Besprechung dieses Phänomens im Rahmen der schulischen (universitären) Bildung, was den Weg zur konstruktiven Reflexion über den Sinn des transkulturellen Lernens und seiner Methoden ebnet.

\section{TRANSKULTURELLES LERNEN MIT DEM MEDIUM „FILM“ IN FREMDSPRACHENDIDAKTISCHEN KONTEXTEN}

Im gegenwärtigen wissenschaftlichen Diskurs stehen Phänomene der Inter-, Trans- bzw. Hyperkulturalität hoch im Kurs. Vor allem die beiden ersten Konzepte sorgen innerhalb der Geisteswissenschaften (u.a. in der Kulturwissenschaft, Anthropologie, interkulturellen Kommunikation, Pädagogik, Literaturdidaktik, Fremdsprachendidaktik) für rege Diskussionen zu terminologischen Bestimmungen und Abgrenzungsversuchen, was angesichts der Tatsache, dass sogar der ihnen zugrunde liegende Terminus „Kultur" sehr breit und vielfältig begriffen wird (vgl. Kepser 2015: 77), nicht wundert. Da in dem vorliegenden Beitrag der Begriff "transkulturell“ mit wissenschaftlich-didaktischer Intention durchgehend verwendet wird, soll das „vorherrschende[] Paradigma“" (Dawidowski 2015: 17) der Interkulturalität von der hier im Fokus stehenden Transkulturalität definitorisch unterschieden werden. 
Die Initiierung der Debatte rund um Transkulturalität wird gewöhnlich Wolfgang Welsch zugeschrieben, obwohl Iljassova-Morger (2009: 38) bemerkt, dass er weder "der erste [noch] der einzige [ist], der auf die neuen transkulturellen Prozesse aufmerksam gemacht hat" und dass sowohl Ulf Hannerz mit seinem kulturanthropologischen Konzept der "globalen Ökumene" als auch Elmar Holenstein mit seinem Verständnis der Kulturen als dynamischen und eng miteinander verflochtenen Gebilden als Vorläufer der Transkulturalität gelten können. All diesen Wissenschaftlern ist gemeinsam, dass sie sich von dem eine Grundlage für die Interkulturalität bildenden Herder'schen Kulturkonzept distanzieren. Im Herder'schen „metaphorische[n] Kugelmodell der Kulturen [...] handelt [es] sich um in sich abgeschlossene Räume, die durch Korridore miteinander verbunden sind oder sich zumindest verbinden lassen" (Kepser 2015: 81). Aus diesem Grund wird in dem Interkulturalitätskonzept oft die Raummetapher verwendet (Kulturkreise, Kulturräume), und es wird angestrebt, Verständigung zwischen unterschiedlichen Kulturen durch eine grenzüberschreitende Kommunikation zu erreichen (vgl. Schumann 2008: 83). Zentral ist dabei die Vorstellung, dass klar umrissene Grenzen zwischen „uns“ und den "Anderen“, bzw. dem Eigenen und dem Fremden zu verzeichnen sind, die überwunden werden müssen, um das Fremdverstehen (vgl. Bredella, Meißner, Nünning \& Rösler 2000) zu ermöglichen (vgl. Schumann 2008: 83). Es war zum großen Teil eben der "klassische[], holistische[], obsolet gewordene Kulturbegriff“ (Iljassova-Morger 2009: 39) innerhalb des Interkulturalitätskonzeptes sowie die Inszenierung der (inter)kulturellen Kontakte als ein Aufeinandertreffen von separaten und homogenischen Welten, die den Grundstein für die Entwicklung einer alternativen Idee der Transkulturalität legte. Welsch (2000) kritisierte sowohl Interkulturalität als auch Multikulturalität dafür, dass sie

Kulturen als autonome, voneinander abgegrenzte Sphären darstellen und einzelne Individuen auf ihre nationalkulturelle Zugehörigkeit reduzieren, was sowohl deskriptiv als auch normativ verfehlt sei, so manche Lebensweisen ignoriere und sogar zu Separatismus, Gettoisierung oder im schlimmsten Fall zu kulturellem Rassismus tendiere (Iljassova-Morger 2009: 39).

So plädierte er für den Transkulturalitätsansatz, der dem aktuellen Zustand der Verzahnung, Überlappung und Durchdringung der Kulturen Rechnung trägt und somit die kultur-gesellschaftlichen Folgen der verstärkten Globalisierungsprozesse, darunter die Hybridisierung der Kulturen (vgl. Wintersteiner 2010), besser aufgreift.

Jedoch findet auch die Transkulturalität ihre Kritiker. Gegen diesen Ansatz wird vor allem eingewendet, dass in ihm paradoxerweise auf das kritisierte Kulturkonzept zurückgegriffen wird (vgl. Iljassova-Morger 2009: 41), 
dass er die Gefahr birgt, „die Macht der Tradition zu unterschätzen, indem [er] das Schwinden der Bedeutung von Nationalstaatlichkeit und Muttersprachlichkeit behaupte" (Dawidowski 2015: 25) und dass in seinem Rahmen kulturelle, politische und ökonomische Asymmetrien und Machtverhältnisse zwischen kulturellem Zentrum und Peripherien nivelliert werden (vgl. Iljassova-Morger 2009: 43). All diese Vorwürfe sollen jedoch nach Kepser (2015: 82) nicht zur pauschalen Ablehnung des Konzeptes führen, sondern als "mahnendes Korrektiv verstanden werden" (Kepser 2015: 82). Interkulturalität und Transkulturalität sollten außerdem nicht als Oppositionspaar begriffen werden, denn es handelt sich um "komplementierende Vorstellungen“, da "die Transkulturalität den Gegenstand der Erkenntnis ${ }^{1}$ und die Interkulturalität das Verfahren der Erkenntnis darstellt" (Schumann 2008: 83-84).

Ähnlich wie Interkulturalität und Transkulturalität sind auch das interund transkulturelle Lernen als einander ergänzend zu betrachten. Sie weisen viele Parallelen auf und die Unterschiede zwischen ihnen verlaufen entlang ähnlicher Linien wie diejenigen zwischen der Inter- und Transkulturalität:

Während interkulturelles Lernen vor allem auf dialogische Verständigungsprozesse zwischen dem Eigenen und dem Fremden - realer oder symbolisch-medialer - Natur zielt, sind Dialogizität und Durchlässigkeit in einem transkulturellen Kulturverständnis schon weitaus stärker mitgedacht und eröffnen konsequenterweise neue Dialoge zwischen verschiedenen Lebens- und Arbeitswelten, innerhalb oder zwischen kulturellen Räumen (Globalität \& Lokalität) (Blell \& Rust 2012: 114).

Laut Welsch wurden im Zentrum polis des Bundesministeriums für Bildung folgende Merkmale und Ziele des transkulturellen Lernens festgelegt:

- Sensibilität für Gleichheit und Differenz, für Anderssein und Gemeinsamkeiten entwickeln;

- Hinterfragen der Entwicklung von individuellen und kollektiven Identitäten;

- Reflexion zur eigenen Position in der Gesellschaft: Was macht mich/uns aus?

- die eigene kulturelle Selbstreflexion durch die Auseinandersetzung mit einer Vielfalt an kulturellen Differenzen stärken;

- eine anerkennende, fragende und lernende Haltung einnehmen;

- kulturelle Vielfalt im Zusammenhang mit gesellschaftlicher Teilhabe, struktureller Benachteiligung und Rassismus-Erfahrungen wahrnehmen;

- Diversität und Heterogenität als Bereicherung anerkennen;

(Welsch 2005, nachfolgend Zentrum polis des Bundesministeriums für Bildung 2016: 6).

${ }^{1}$ Hier geht es u.a. um transkulturelle Lebensläufe der MigrantInnen, die auf eine transkulturelle Weise in ausgewählten Culture-Clash-Komödien porträtiert werden. 
Die so formulierten Ziele des transkulturellen Lernens basieren auf der Annahme der kulturellen Pluralität der menschlichen Identitäten und der Tatsache, dass ein "ständiger kultureller Austausch der Normalfall ist" (Kepser 2015: 82), was in den im vorliegenden Beitrag nachstehend analysierten Culture-Clash-Komödien deutlich zum Ausdruck kommt.

Das transkulturelle Lernen kann dann sowohl selbst- als auch fremdinitiiert sein (vgl. Kepser 2015: 82) - auch mit Hilfe unterschiedlicher Medien, wie z.B. des Filmes. Das (fremdsprachen)didaktische Potential des Filmes bei der Förderung des transkulturellen Lernens ergibt sich u.a. aus den folgenden Merkmalen dieses Mediums:

1. Geschichtliche Entwicklung des Filmes: in seinen Anfängen war der Film stumm und die SchauspielerInnen mussten sich in ihrer Arbeit vor allem der Mittel der nonverbalen Kommunikation bedienen. Die nonverbale Kommunikation ist weniger kulturspezifisch als die verbale und wird oft "intuitiv verstanden“ (Kepser 2015: 84), was den Transfer bestimmter Inhalte und Botschaften über die kulturellen Grenzen hinaus erleichtert. Überdies bemerkt Kepser (2015: 84), dass die Geburtsstunde des Filmes mit der fortschreitenden technologischen Entwicklung (z.B. Erfindung des Telefons, des Autos, des Flugzeugs) einherging, die wiederum die Globalisierungsprozesse angetrieben hat. Aus diesem Grund kann der Film als ein von seinen Ursprüngen her globales Medium bezeichnet werden.

2. Kulturdidaktisches Potential des Filmes: durch das Eintauchen in die filmische Realität wird auch der Kontakt mit einer fremdsprachigen Kultur ermöglicht. Diese wird im Film durch Sprache, Thematik, Verhaltensweisen der ProtagonistInnen und Figuren, unterschiedliche private und öffentliche Räume, linguistic landscapes, einzelne Gegenstände u.v.m. sichtbar, erlebbar bzw. vermittelbar. Hier sind nicht nur ein Film als solcher, sondern u.a. auch Aspekte der Filmproduktion (z.B. internationale Koproduktionen) und der Filmdistribution (z.B. wechselnde Filmtitel) zu beachten (vgl. Badstübner-Kizik 2014: 77-78).

3. Thematik des Filmes: Kepser (2015: 88) konstatiert: „Am offensichtlichsten kann ein Film dann transkulturell bedeutsam sein, wenn er transkulturelle Begegnungen direkt thematisiert und damit die $\mathrm{Zu}$ schauer konfrontiert". $\mathrm{Zu}$ den Themen, die eine kritische Auseinandersetzung mit (trans)kulturellen Themen anregen können, gehören u.a. kulturelle Auto- und Heterostereotype, kulturelle Herrschaftsbzw. Dominanzverhältnisse, hybride Identitäten, Migrationserfahrungen, Zusammenhang von Sprache und (kultureller) Identität usw. (vgl. Kepser 2015: 97-98). 
4. Multimedialität des Filmes und die damit verbundene Fülle an didaktisch-methodischen Angeboten: Filme setzten sich aus audiovisuellen, auditiv-akustischen, textuellen Formaten zusammen und sind eng verbunden mit anderen schriftlichen, auditiven und audiovisuellen Medien, wie z.B. mit Filmplakaten, Filmmusik, Interviews mit u.a. SchauspielerInnen, RegisseurInnen und DrehbuchautorInnen, Rezensionen, Berichten, Blogs, Filmportalen usw. Dadurch können den Lernenden unzählige handlungsorientierte, kreative Aufgaben zur Förderung von Hör-Sehverstehen, Hörverstehen, Leseverstehen, mündlicher und schriftlicher Kommunikationsfähigkeit sowie zur Unterstützung interkultureller Kompetenz angeboten werden, die sich entweder auf den ganzen Film, einzelne Auszüge oder konkrete Szenen sowie alle anderen begleitenden Medien beziehen und folgende Aktivitäten umfassen, wie u.a. Vermutungen anstellen, recherchieren, Assoziationen sammeln und Assoziogramme erstellen, Zusammenfassung / Rezension / alternatives Ende schreiben, eigene Filmszenen drehen u.v.m. (vgl. Badstübner-Kizik 2014: 76).

5. Mehrsprachigkeit des Filmes: auch wenn der Film selbst nicht als mehrsprachig bezeichnet werden kann, dann sind seine Produktion, Distribution und Rezeption i.d.R. doch mehrsprachig. Filme werden exportiert und übersetzt, sie haben eine Internetpräsenz in unterschiedlichen Sprachen, es werden Rezensionen über die Filme in verschiedenen Sprachen verfasst (vgl. Badstübner-Kizik 2014: 78). All diese Erscheinungen können zum Gegenstand einer sprachbewussten und kulturaufmerksamen Analyse bei transkulturellen Lernprozessen werden.

Viele von den oben beschriebenen Charakteristika (z.B. die (trans)kulturelle Thematik oder Mehrsprachigkeit) weisen Culture-Clash-Komödien auf, deren (fremdsprachen)didaktisches Potential als Katalysator des transkulturellen Lernens wirken kann.

\section{CULTURE-CLASH-KOMÖDIEN ALS TEIL DER TRANSKULTURELLEN KINEMATOGRAPHIE}

Der Begriff "Culture-Clash-Komödie“, im englischsprachigen Raum vor allem als "fish-out-of-water-comedy" bezeichnet, bezieht sich nach Rösch (2015: 193) auf eine „Filmkomödie, die die Begegnung oder auch den Zusammenprall verschiedener Kulturen satirisch überzeichnet oder grotesk verfremdet darstellt". Die Komik, vor allem die Situationskomik, die in solchem Subgenre der Filmkomödie von zentraler Bedeutung ist, speist sich „in 
der Regel [aus] interkulturellen Missverständnissen, weil die Äußerungsformen der je anderen Kultur unbekannt sind, ignoriert oder missachtet werden" (Rösch 2015: 193). Die Typen- und Figurenkomik basiert vorwiegend auf Stereotypen und kulturellen Besonderheiten (vgl. Rösch 2015: 193). Culture-Clash-Komödien sind eine relativ neue Erscheinung im sog. transnationalen $\mathrm{Kino}^{2}$, zumindest in Deutschland ${ }^{3}$ - je nach Land und Geschichte seiner Migration bzw. Geschichte der cineastischen Darstellung der Migrationserfahrungen gibt es europa- und weltweite Unterschiede.

In Deutschland werden in Bezug auf das transnationale Kino gewöhnlich folgende Entwicklungsphasen ausgesondert: „Phase der Betroffenheit, Opferfiguren und Fragen nach der Identität an einem neuen Aufenthaltsort, Phase der interkulturellen Themen [...], Phase der transkulturellen Themen und humoristischen Darstellung" (Chmielewska 2014: 132). Die erste Phase zeichnete sich dadurch aus, dass es vor allem die Deutschen waren, die hinter die Kamera traten, um über das harte MigrantInnenleben zu erzählen und somit zu politischen und sozialen Phänomenen mitfühlend Stellung zu nehmen (vgl. Chmielewska 2014: 132). In der zweiten Phase wurde die Regie der Filme zur Migration öfter von Menschen geführt, die selber einen Migrationshintergrund hatten. Die thematischen Schwerpunkte blieben denjenigen aus der ersten Phase ähnlich, d.h. die Zerrissenheit zwischen zwei kulturellen Welten oder die gesellschaftliche Stellung der Frauen mit Migrationshintergrund (vgl. Chmielewska 2014: 132). Culture-Clash-Komödien gehören in die letzte Phase, also in die Phase der Transkulturalität und der humorvollen Darstellungsweise, deren Beginn ungefähr auf die 1990er Jahre zu datieren ist (und die bis heute anhält). Seit dieser Zeit sind viele populäre Filme entstanden, die diesem Genre angehören, wie z.B. "Alles getürkt" (Reg. Yasemin Şamdereli, Deutschland 2003), „Kebab Connection“ (Reg. Anno Saul, Deutschland 2005), „Salami Aleikum" (Reg. Ali Samadi Ahadi, Deutschland 2009), „Soul Kitchen“ (Reg. Fatih Akin, Deutschland / Frankreich 2009), „Polnische Ostern“ (Reg. Jakob Ziemnicki, Deutschland 2011) oder "Almanya - Willkommen in Deutschland“ (Reg. Yasemin Şamdereli, Deutschland 2011). ${ }^{4}$ Kepser (2015: 89) bemerkt, dass die meisten Culture-

\footnotetext{
${ }^{2}$ Mehr zur Entwicklung der Terminologie vom „Migrantenkino“ bis "cinema of transvergance" bei Chmielewska (2014).

${ }^{3}$ Weil wir zwar nicht ausschließlich, wie die Beispiele für Culture-Clash-Komödien im weiteren Teil des vorliegenden Beitrags zeigen, jedoch vorwiegend mit Culture-ClashKomödien aus Deutschland und in germanistischen Bildungskontexten arbeiten, werden hier nur eben diese kurz thematisiert.

${ }^{4}$ Mehr Beispiele für Culture-Clash-Komödien aus Deutschland unter https://www.phkarlsruhe.de/fileadmin/user_upload/hochschule/masterstudimm/Liste_CultureClash_Komo edien.pdf (Zugriff am: 28.08.2018).
} 
Clash-Komödien nicht als „Verlachkomödien“, sondern als „Aufklärungskomödien" zu verstehen sind, von denen eine positive Botschaft im Sinne der transkulturellen Bildung hervorgeht und zwar, dass die (kulturellen, sprachlichen, sozialen) Unterschiede nicht kaschiert werden sollen sowie die von ihnen verursachten Konflikte lösbar sind und ein „verträgliches $\mathrm{Zu}$ sammenleben“" (Kepser 2015: 89) nicht verhindern müssen. Die in ihnen porträtierten transkulturellen Biographien und Identitäten laden zur Reflexion über die gesellschaftliche Gleichheit und Beteiligung sowie die Gemeinsamkeiten zwischen den Menschen ein, was wiederum eine Basis des transkulturellen Lernens ausmacht.

\section{INTERSEKTIONELLE ANALYSE DER MIGRANTINNENBILDER IN AUSGEWÄHLTEN CULTURE-CLASH-KOMÖDIEN ALS BEISPIEL DES TRANSKULTURELLEN LERNENS}

Beispiele für Culture-Clash-Komödien, die sich als didaktische Vorlagen für transkulturell ausgerichtete Lernkontexte gut eignen, sind die drei folgenden Filme: „Lost in Translation“ (Reg. Sofia Coppola, USA 2003), „Willkommen bei den Sch'tis“ (Reg. Dany Boon, Frankreich 2008) und „Almanya - Willkommen in Deutschland“ (Reg. Yasemin Şamdereli, Deutschland 2011). Sie wurden in folgenden Lehrveranstaltungen und mit folgenden Zielgruppen eingesetzt:

1. „Lost in Translation“: Auszüge aus dem Film wurden in Cultural Awareness Training mit Studierenden der Angewandten Linguistik der Adam-Mickiewicz-Universität Poznań, in der Lehrveranstaltung „Polen und Deutsche in Europa" mit den Studierenden des Masterstudienganges „Interkulturelle Studien: Polen und Deutsche in Europa" an der Christian-Albrechts-Universität zu Kiel sowie in einem interkulturellen Training mit polnischen und deutschen Studierenden als Einstieg in das Thema „Übersetzbarkeit von Sprachen und Kulturen" und "Kulturschock" präsentiert.

2. „Willkommen bei den Sch'tis“: gezeigt wurde der ganze Film in einem interkulturellen Training sowohl für eine polnische als auch deutschpolnische Studierendengruppe zur Illustration und Analyse solcher Fragestellungen, wie "Was sind Auto- und Heterostereotype?", „Welche Funktionen erfüllen Stereotype?", „Was sind Mechanismen der Entstehung der Stereotype und was kann zu ihrer Relativierung beitragen?"

3. „Almanya - Willkommen in Deutschland“: Projektion des ganzen Filmes erfolgte in der Lehrveranstaltung „Interkulturelle Literatur" 
mit Studierenden der Angewandten Linguistik der Adam-MickiewiczUniversität Poznań als Einführung in die Hauptthemen des Seminars, wie z.B. Geschichte der Arbeitsmigration nach Deutschland nach 1945, die erste, zweite, dritte Generation der Menschen mit Migrationshintergrund, individuelle und gesellschaftliche Folgen der Migration, hybride Identitäten.

Es wurde versucht, die bereits besprochenen Dimensionen / Themen / Arbeitsfelder Multimedialität, Mehrsprachigkeit und transkulturelles Potential der Filme durch mediendidaktische Maßnahmen hervorzuheben. Diese umfassten entsprechende Aufgabenstellungen, die sowohl die künstlerische bzw. ästhetische als auch die inhaltliche Seite des Mediums berücksichtigen sollten. Den Studierenden wurden u.a. folgende Aufgaben zur Erschließung der Themen und ihren Darstellungsformen in den hier besprochenen Filmen gestellt:

a. zu „Lost in Translation“: (1) Schlagen Sie bitte einen alternativen polnischen bzw. deutschen Titel für den Film vor! Überlegen Sie dabei, was in der Szene als „in der Translation verloren gegangen“ gemeint ist! (2) Machen Sie bitte Notizen zu den in der Szene eingesetzten filmtechnischen Mitteln! Wie ist die Beleuchtung? Werden alle Gegenstände und Personen gleichermaßen beleuchtet? Warum (nicht)? Wie sind die Kameraführung, die Einstellungsgrößen und der Ton? Wie wirken Sie auf Sie und in welchem Lichte stellen sie den Regisseur, die Dolmetscherin und den Schauspieler in der Szene dar? (3) Welche in der Szene erwähnten Begriffe können als kulturspezifische Phänomene wahrgenommen werden?

b. zu "Willkommen bei den Sch'tis“: (1) Schauen Sie sich das Plakat an und lesen Sie die Kurzbeschreibung des Filmes! Zu welchem Genre gehört der Film? Was erwarten Sie von ihm in Bezug auf Inhalt? (2) Notieren Sie bitte, welche Szenen sich konkret auf folgende Aspekte beziehen: Autostereotype, Relativierung von Stereotypen, Funktionen von Stereotypen!

c. zu "Almanya - Willkommen in Deutschland“: (1) Wie hat Ihnen der Film gefallen? Charakterisieren Sie ihn bitte spontan mit Hilfe von drei Adjektiven! (2) Was sind die Hauptthemen des Filmes? Sammeln Sie diese bitte in Stichpunkten!

Vor dem Hintergrund eines medialen Bildes von MigrantInnen möchten wir jedoch von der inhaltlichen Seite her eine andere Aufgabe vorstellen, die eben zur Analyse der Darstellungsformen von MigrantInnen in ausgewählten literarischen und filmischen Werken im Seminar "Interkulturelle Literatur" eingesetzt wurde. Das Ziel der Aufgabe ist es, durch die Arbeit mit transkulturellen Filmen „verschiedene Dimensionen von Differenz und He- 
terogenität zu verdeutlichen" (Holzwarth 2013) und somit dem Konzept der Intersektionalität gerecht zu werden, das ein „Paradigma“ bedeutet,

mit dem die Wechselbeziehungen von Dimensionen sozialer Macht-, Herrschafts- und Normierungsverhältnisse wie Geschlecht, soziales Milieu, Migrationshintergrund, Nation, Ethnizität, „Rasse“, sexuelle Orientierung, Behinderung, Generation et_cet_era. fokussiert werden. Diese Dimensionen sozialer Ungleichheit werden als soziale Konstruktionen konzeptualisiert, welche nicht isoliert voneinander analysiert werden können, sondern in ihren Überschneidungen (intersections) oder Wechselverhältnissen (Interdependenzen / interdependenten Kategorien) untersucht werden müssen (http://portal-intersektiona litaet.de/konzept/, nachfolgend Holzwarth 2013).

Dank solch einer intersektionellen Analyse soll der - wie Holzwarth (2013) bemerkt - kontraproduktiven Unterscheidung in Migrant - Nicht-Migrant entgegengewirkt werden.

Im Seminar wurden als Grundlage für die intersektionelle Analyse und den Vergleich des MigrantInnenbildes folgende Szenen aus den drei Filmen gezeigt:

a. „Lost in Translation“: es wurde ein Filmausschnitt gewählt, in dem der Protagonist, ein US-amerikanischer Schauspieler, eine Werbung für Whisky in Japan drehen soll. In der Szene erzählt der japanische Regisseur dem Schauspieler auf Japanisch von seiner Vision der Werbung, erklärt den Kontext der Werbung und gibt ihm konkrete Anweisungen. All diese Informationen sollen von der Dolmetscherin ins Englische übertragen werden, was nur z.T. geschieht und zu komischen Missverständnissen führt. ${ }^{5}$

b. „Willkommen bei den Sch'tis“: in der untersuchten Szene unterhält sich der aus dem Zentrum Frankreichs stammende Postleiter mit seinem Mitarbeiter, einem Sch'ti, also einem Nordfranzosen, und deutet dabei falsch die Formulierung "und sage Ihnen was", was fast zur Ursache eines Konflikts zwischen den beiden wird. Erst die Erklärungen einer anderen Mitarbeiterin des Postamtes helfen dabei, ein sprachliches Missverständnis zu klären. Das kurze und komische Gespräch verläuft folgendermaßen:

Postleiter: Antoine, das muss dringend zum Chef des Briefzentrums, der Leiter wartet schon darauf. Sobald Sie dort sind, geben Sie mir Bescheid.

Antoine: Verstehe, ich ruf Sie an und sag Ihnen was.

${ }^{5}$ Diese Szene ist unter https://www.youtube.com/watch?v=gXGXZiX0pCA zugänglich (Zugriff am: 28.08.218). 
Postleiter: $\quad \mathrm{Na}$, dass er die Unterlagen bekommen hat.

Antoine: Ja, genau. Ich ruf Sie an und sage was.

Postleiter: Das „was“ habe ich Ihnen gerade doch gesagt.

Antoine: Ja, und ich hab's verstanden.

Postleiter: Sie rufen mich also an?

Antoine: Ja... Ich übergebe es und ruf Sie von dort aus an und sag Ihnen was.

Postleiter: Tja, was weiß ich denn. Zum Beispiel „Hallo, hier ist Antoine. Ich hab die Sendung gerade übergeben“. Sowas in der Art. Alles klar?

Antoine: Ja, ich bin kein Dödel. Ich hab's verstanden.

Postleiter: Gut, dann rufen Sie mich an.

Antoine: Und sag Ihnen was.

Postleiter: Es reicht! Sie sind betrunken!

Antoine: Nein!

Annabelle: Nein, nein, Herr Direktor. Das „sag Ihnen was“ ist Sch'ti und bedeutet so viel wie "ich sage Ihnen Bescheid.“

Postleiter: Ach, verstehe! Tut mir Leid.

Antoine: Nix passiert, ja.

Postleiter: Sie rufen mich also an und sagen was.

Antoine: $\quad \mathrm{Na}$, dass er die Unterlagen hat, oder?

(00:50':14"- 00:51':10")

c. "Almanya - Willkommen in Deutschland": die Szene spielt in den 1970er Jahren in Deutschland. Man sieht eine junge Türkin, Fatima, die in einen Lebensmittelladen kommt, um Einkäufe zu machen. Da sie aber kein Wort Deutsch spricht und es sich um keinen Selbstbedienungsladen handelt, muss sie mit dem Verkäufer ins Gespräch kommen, um zu erklären, was sie braucht. Fatima und der Verkäufer müssen sich vorwiegend nonverbal verständigen, was lustige, auch kulturbedingte Missverständnisse provoziert. ${ }^{6}$

Die präsentierten Filmausschnitte wurden in drei Schritten analysiert:

1. Zuerst wurden die grundlegenden Unterschiede in der Situation der MigrantInnen herausgearbeitet. Es wurde nach dem Geschlecht, dem Ziel- und Herkunftsland sowie dem Grund der Migration der Filmfiguren gefragt. Bei „Lost in Translation“ handelt es sich um einen amerikanischen Schauspieler, der für kurze Zeit nach Japan kommt, um dort eine Werbung zu drehen. Im Film „Willkommen bei den Sch'tis" geht es ebenfalls um einen Mann und eine Arbeitsmigration, diesmal aber innerhalb desselben Landes. Der Protagonist wird in den Norden Frankreichs versetzt und hat dort die Stellung eines Postleiters inne.

${ }^{6}$ Diese Filmsequenz ist unter https://www.youtube.com/watch?v=riXvVkhU-2A zu sehen (Zugriff am: 28.08.2018). 
Im Fall von Fatima aus "Almanya - Willkommen in Deutschland" geht es um eine Frau aus der Türkei, die in den 1970er Jahren im Rahmen des Familiennachzugs als Frau eines Gastarbeiters nach Deutschland kommt. Diese grundsätzlichen Unterschiedlichkeiten in der sozialen Position zwischen den FilmprotagonistInnen sind für den nächsten Punkt der Reflexion grundlegend.

2. Im zweiten Schritt wurden die Studierenden gebeten, einen gemeinsamen Nenner für die dargestellten Szenen zu finden, der spontan als "Kommunikationsprobleme" identifiziert wurde. Und eben das Prisma des Zugangs zur Sprache bzw. Übersetzung / Verdolmetschung und somit zur Teilhabe an Kultur und bestimmten Diskursen bildet die zentrale Perspektive für die Analyse der MigrantInnenbilder in den hier besprochenen Filmen. Zum Leitmotiv der Überlegungen wird die in dem Essay von der Literaturwissenschaftlerin Gayatri Chakravorty Spivak (1988) gestellte Frage "Can the Subaltern Speak?", wobei "subaltern" hier als MigrantInnen verstanden wird. Die SeminarteilnehmerInnen untersuchen, ob und wie die kommunikativen Absichten der MigrantInnen realisiert werden. Der Schauspieler aus „Lost in Translation" hätte aufgrund seines sozialen Status - er ist ein reicher, erfolgreicher Mann aus den Vereinigten Staaten, der für berufliche Zwecke nach Japan gekommen ist - die Möglichkeit, sich dank der Verdolmetschung mit dem Werbungsregisseur zu verständigen. Das Missverständnis und die damit einhergehende Komik resultieren aus der Tatsache, dass die Dolmetscherin ihre Rolle auf die Tradierung der sprachlichen Inhalte reduziert und ihre Aufgabe als Kulturmittlerin ignoriert. Trotz der Nachfragen des Schauspielers erklärt sie nicht, warum die Verdolmetschung viel kürzer ist, als die Aussagen des Regisseurs und was für den japanischen Regisseur „more intensity“ bedeuten könnte. Die misslungene Kommunikation ist also nicht durch den Migrationsstatus des Schauspielers bedingt, denn ihm stehen mutmaßlich bestimmte Ressourcen zur Verfügung, die jedoch ungenutzt bleiben. Auch der Postleiter aus "Willkommen bei den Sch'tis“ hat die Möglichkeit, nach einer Verdolmetschung zu bitten. Er ist auch ein Arbeitsmigrant, der etwas nicht versteht, aber seine kommunikative Lage ist durch zwei Faktoren erleichtert: erstens, er und sein Gesprächspartner sprechen eine gemeinsame Sprache, Französisch, nur in zwei Varietäten, und zweitens ist er der Chef seines Gesprächspartners, wodurch sein Unverständnis einer bestimmten Formulierung nicht so leicht ignoriert werden kann. Am Rande soll hier bemerkt werden, dass es in der Szene aus „Willkommen bei den Sch'tis“ zu einer interessanten Umkehrung der Situation in Bezug auf die Sprache 
kommt. Normalerweise werden diejenigen aus der Kommunikation ausgeschlossen, die z.B. sog. gebrochene Sprache oder Migrantensprache, also keine Standardsprache sprechen. Hier hat sich die Unkenntnis des Dialektes als Kommunikationshürde erwiesen. In der letzten Szene, aus dem Film "Almanya - Willkommen in Deutschland“, wurde - filmtechnisch gesehen - ein zusätzlicher Verfremdungseffekt erreicht, dadurch dass Fatima Deutsch und der Verkäufer Türkisch sprechen. Im Vergleich zum Schauspieler und Postleiter aus den zwei vorherigen Filmen verfügt Fatima, als Frau aus der Türkei, über keine Macht und Ressourcen. Sie ist auf ihre Kreativität in der Kommunikation sowie auf den guten Willen des Verkäufers angewiesen. Sie ist in der Kommunikation nur zum Teil erfolgreich. Die fehlenden Sprachkenntnisse und die von dem Verkäufer falsch gedeuteten Gesten hindern sie daran, Brot zu kaufen. Fatima gelingt es aber, Milch zu kaufen und dabei ein neues Wort aus der ihr fremden Sprache zu lernen, das sie mit großer Freude und Erleichterung in ihr mentales Lexikon aufnimmt.

3. Im dritten und letzten Schritt sollten die Studierenden die im zweiten Punkt gestellte Frage beantworten, ob und unter welchen Bedingungen man "subaltern" - hier auf Seiten der MigrantInnen - sprechen kann. Zudem sollten sie zusammenfassend reflektieren, wie sich der soziale Status der MigrantInnen auf die Mechanismen der interkulturellen Kommunikation auswirkt. Dank der Analyse des MigrantInnenbildes in seiner Intersektionalität bzw. in interdependenten Kategorien, erfahren die SeminarteilnehmerInnen, dass es bei dem Versuch, diese Fragen zu beantworten, nicht genügt zu überlegen, was es heißt, MigrantIn oder Nicht-MigrantIn zu sein, sondern man muss immer mitbedenken, was es heißt - auf den hier präsentierten Beispielen basierend - ein Mann, Chef, erfolgreicher Schauspieler und Migrant oder eine Frau ohne Fremdsprachenkenntnisse und Migrantin zu sein. Dadurch wird "einem einseitigen Deuten und Wahrnehmen in ethnischen Kategorien“ kein „Vorschub geleistet“ (Holzwarth 2013).

\section{FAZIT}

Der oben beschriebene Vorschlag für die Auseinandersetzung mit dem MigrantInnenbild erfolgte mit dem Ziel, Prozesse der transkulturellen Bildung zu initiieren und zu fördern sowie didaktische Wirksamkeitspotentiale des Mediums „Film“ bei der Nuancierung der MigrantInnenbilder aufzuzeigen. Als Zusatzfaktor wurden auch die Herrschaftsverhältnisse analy- 
siert, die sich im Zugang zur und Verwendung von einer bestimmten Sprache manifestieren sowie die Möglichkeit der gesellschaftlichen Teilhabe in Relation zur sozialen und materiellen Situation der MigrantInnen beeinflussen. Was anfänglich als Aufmerksamkeit und Interesse weckender Unterhaltungsfaktor eingeordnet werden konnte, entwickelte sich womöglich zum didaktischen Anreiz, der dank dem Einsatz angemessener (film)didaktischer Methoden eine Brücke zwischen Spaß und vertiefter Analyse geschlagen hat. Durch das hier angestrebte inter- und transkulturelle Lernen sollen die Lernenden dafür sensibilisiert werden, kein verallgemeinertes MigrantInnenbild zu konstruieren, sondern u.a. die soziologischen, (national)kulturellen und linguistischen Aspekte bei der Individualisierung der MigrantInnenbilder zu berücksichtigen.

\section{LITERATURVERZEICHNIS}

Badstübner-Kizik, C. (2014). Erfolg in authentischen fremdsprachigen Lernräumen? Das Beispiel „Film“. In: J. Sujecka-Zając / A. Jaroszewska / K. Szymankiewicz / J. Sobańska-Jędrych (Hrsg.), Inspiracja - Motywacja - Sukces. Rola materiałów dydaktycznych i form pracy na lekcji jezyka obcego (S. 71-88). Warszawa: Instytut Germanistyki Uniwersytetu Warszawskiego i Instytut Romanistyki Uniwersytetu Warszawskiego.

Blell, G. / Rust, I. (2012). E-Begegnungen und transkulturelles Lernen in der literaturdidaktischen Ausbildung von Lehramtsstudierenden. In: G. Blell / Ch. Lütge (Hrsg.), Fremdsprachendidaktik und Lehrerbildung: Konzepte, Impulse, Perspektiven (S. 113-131). Berlin: LIT Verlag.

Bredella, L. / Meißner, F.-J. / Nünning, A. / Rösler, D. (Hrsg.) (2000). Wie ist Fremdverstehen lehr- und lernbar? Vorträge aus dem Graduiertenkolleg "Didaktik des Fremdverstehens". Tübingen: Gunter Narr Verlag.

Chmielewska, K. (2014). Von Angst essen Seele auf bis Almanya - deutsch-türkische Filme im interkulturellen Fremdsprachenunterricht. Glottodidactica, XL (1), 127-139.

Dawidowski, Ch. (2015). Inter-, Transkulturalität und Literaturdidaktik. Einführender Forschungsüberblick. In: Ch. Dawidowski / A. R. Hoffmann / B. Walter (Hrsg.), Interkulturalität und Transkulturalität in Drama, Theater und Film. Literaturwissenschaftliche und didaktische Perspektiven (S. 17-41). Frankfurt am Main: Peter Lang.

Holzwarth, P. (2013). Filmliste Migration. https://www.ph-ludwigsburg.de/fileadmin/sub sites/1b-mpxx-t-01/user_files/Online-Magazin/Ausgabe16/Filmliste_Migration16.pdf (Zugriff am: 28.08.2018).

Iljassova-Morger, O. (2009). Transkulturalität als Herausforderung für die Literaturwissenschaft und Literaturdidaktik. Das Wort. Germanistisches Jahrbuch Russland, 37-57.

Kepser, M. (2015). Transkulturelle Bildung mit Film im Deutschunterricht. Eine kulturwissenschaftliche Ergründung des Handlungsfelds. In: Ch. Dawidowski / A. R. Hoffmann / B. Walter (Hrsg.), Interkulturalität und Transkulturalität in Drama, Theater und Film. Literaturwissenschaftliche und-didaktische Perspektiven (S. 77-106). Frankfurt am Main: Peter Lang.

Rösch, H. (2015). Culture-Clash-Komödien im Literaturunterricht der Sekundarstufen. In: Ch. Dawidowski / A. R. Hoffmann / B. Walter (Hrsg.), Interkulturalität und Transkulturalität 
in Drama, Theater und Film. Literaturwissenschaftliche und -didaktische Perspektiven (S. 193-216). Frankfurt am Main: Peter Lang.

Schumann, A. (2008). Transkulturalität in der Romanistischen Literaturdidaktik. Kulturwissenschaftliche Grundlagen und didaktische Konzepte am Beispiel der ,littérature beur'. Fremdsprachen Lehren und Lernen, 37, 81-94.

Spivak, G. Ch. (1988). Can the Subaltern Speak? In: C. Nelson / L. Grossberg (Hrsg.), Marxism and the Interpretation of Culture (S. 271-313). Chicago: University of Illinois Press.

Welsch, W. (2000). Transkulturalität. Zwischen Globalisierung und Partikularisierung. Jahrbuch Deutsch als Fremdsprache, 26, 327-351.

Wintersteiner, W. (2010). Transkulturelle literarische Bildung: Die Poetik der Verschiedenheit in der literaturdidaktischen Praxis. Innsbruck / Wien / Bozen: Studienverlag.

Zentrum polis des Bundesministeriums für Bildung (2016). Transkulturelles und Interkulturelles Lernen. Polis Aktuell, 2. https://www.politik-lernen.at/dl/qOINJMJKomLNIJqx4KJK/ pa_2016_2_trans_interkulturell es_lernen_web.pdf (Zugriff am: 28.08.2018).

Received: 13.09.2018; revised: 27.02.2019 